IRA-International Journal of Applied Sciences ISSN 2455-4499; Vol.04, Issue 01 (2016)

Institute of Research Advances

http://research-advances.org/index.php/IRAJAS

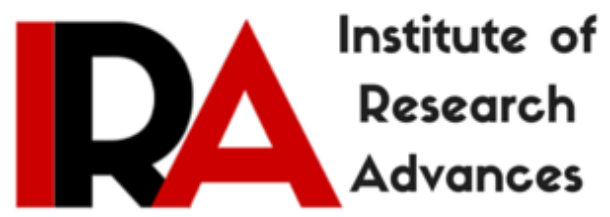

\title{
A Review on Environmentally-safe Corrosion deterrence of Metals in Various Media
}

\author{
R. Vasantha Jothi ${ }^{1}$; R.Saratha ${ }^{1}$; S. V. Priya ${ }^{2}$ \\ ${ }^{1}$ Department of Chemistry, Avinashilingam Institute for Home Science and \\ Higher Education for Women University, Coimbatore - 43, Tamil Nadu, India \\ ${ }^{2}$ Department of Chemistry, Centre for Environmental Research, Kongu \\ Engineering College, Perundurai, Tamil Nadu, India.
}

DOI: http://dx.doi.org/10.21013/jas.v4.n1.p6

\section{How to cite this paper:}

Jothi, R., Saratha, R., \& Priya, S. (2016). A Review on Environmentally-safe Corrosion deterrence of Metals in Various Media. IRA-International Journal of Applied Sciences (ISSN 2455-4499), 4(1). doi:http://dx.doi.org/10.21013/jas.v4.n1.p6

(C) Institute of Research Advances

\section{(cc) EY-NC}

This works is licensed under a Creative Commons Attribution-Non Commercial 4.0 International License subject to proper citation to the publication source of the work.

Disclaimer: The scholarly papers as reviewed and published by the Institute of Research Advances (IRA) are the views and opinions of their respective authors and are not the views or opinions of the IRA. The IRA disclaims of any harm or loss caused due to the published content to any party. 


\section{ABSTRACT}

The present review gives a brief application of environmentally - safe corrosion inhibitors for various metals like aluminum, copper, mild steel, and zinc. Due to the enormous increase of industrial activities, a large amount of mineral acids are consumed. Therefore more number of metals gets corroded. These problems can be solved by using corrosion deterence. Although many chemical deterence are available most of them are highly virulent. So there is a need to explore a new class of corrosion inhibitors with good inhibition efficiency and that are environmentally - safe. Consequently, it can be achieved by using plant materials as corrosion inhibitors which are non-toxic, easily available and biodegradable.

Key words: Aluminum, Mild steel, copper, Zinc, Eco-friendly corrosion inhibitors, plant extract

\section{Introduction:}

Recently, a lot of investigations have been carried out in search of different types of corrosion inhibitors for various types of metals, in different media. Although chemical inhibitors (organic \& inorganic) are available, they cause much damage to our surroundings. Therefore to overcome these problems, the area of research regarding the plant extracts as corrosion inhibitors is being concentrated much because of their renewability, low-cost, eco-friendliness and easy availability. The extract of leaves, stem, bark, root, fruit and seed consists of mixture of organic compounds and has the ability to function as effective inhibitors for metal in acid, alkaline and neutral media. The inhibition process occurs via the phytoconstituents present in the plant material on the surface of the metal, thus a protective film is formed therein corrosion

process

is prevented further. 
A list of plant extracts as eco-friendly corrosion inhibitors is given below:

\begin{tabular}{|c|c|c|c|c|c|c|c|}
\hline S.no & Metal/Medium & & $\begin{array}{l}\text { nhibitor/ } \\
\text { ion parameters }\end{array}$ & Phytochemicals present & Methods adopted & Major findings & $\begin{array}{c}\text { Ref } \\
\text { no }\end{array}$ \\
\hline \multirow[t]{3}{*}{1.} & \multirow{3}{*}{$\begin{array}{l}\text { Aluminum alloy } \\
\text { AA3003/ } \\
0.4 \mathrm{M} \mathrm{HCl} \text { and } \\
0.5 \mathrm{M} \mathrm{HCl}\end{array}$} & \multicolumn{2}{|c|}{ Aspilia africana leaf } & \multirow[t]{3}{*}{ Vitamin B } & \multirow{3}{*}{$\begin{array}{l}\text { Gravimetric, } \\
\text { Electrochemical, } \\
\text { Quantum } \\
\text { chemical } \\
\text { computations \& } \\
\text { Molecular } \\
\text { simulations }\end{array}$} & \multirow{3}{*}{$\begin{array}{l}\text { Both Physisorption and } \\
\text { Chemisorption process } \\
\text { occurred. }\end{array}$} & \multirow[t]{3}{*}{1} \\
\hline & & Conc & $100-700 \mathrm{mg} / \mathrm{L}$ & & & & \\
\hline & & Temp & $303-333 \mathrm{~K}$ & & & & \\
\hline \multirow[t]{3}{*}{2.} & \multirow{3}{*}{$\begin{array}{l}\text { Aluminum/ } 1 \mathrm{M} \\
\mathrm{HCl}\end{array}$} & \multicolumn{2}{|c|}{ Coconut coir dust } & \multirow{3}{*}{$\begin{array}{l}\text { Lignins,Tannins,Cellulose, } \\
\text { Pentosan,Furfural }\end{array}$} & \multirow{3}{*}{$\begin{array}{l}\text { Weight loss and } \\
\text { Hydrogen } \\
\text { evolution methods }\end{array}$} & \multirow{3}{*}{$\begin{array}{l}\text { Chemisorption } \\
\mathrm{IE}-80 \% \text { at } 60^{\circ} \mathrm{C}-\text { Weight loss } \\
\text { method }\end{array}$} & \multirow{3}{*}{2} \\
\hline & & Conc & $0.1-0.5 \mathrm{~g} / \mathrm{L}$ & & & & \\
\hline & & Temp & $30^{\circ} \mathrm{C}$ and $60^{\circ} \mathrm{C}$ & & & & \\
\hline \multirow[t]{3}{*}{3.} & \multirow{3}{*}{$\begin{array}{l}\text { Aluminum/ 2M } \\
\mathrm{HCl}\end{array}$} & \multicolumn{2}{|c|}{ Chlomolaena odorata L. leaf } & \multirow{3}{*}{$\begin{array}{l}\text { Oils,Steroids, Triterpenes, } \\
\text { Flavonoids }\end{array}$} & \multirow{3}{*}{$\begin{array}{l}\text { Gasometric and } \\
\text { Thermometric } \\
\text { techniques }\end{array}$} & \multirow{3}{*}{$\begin{array}{l}\text { Physisorption, } \\
\text { IE }-92.16 \% \text { (Gasometric) } \\
\text { IE }-95.12 \% \text { (Thermometric) at } \\
7.5 \% \text { V/V }\end{array}$} & \multirow[t]{3}{*}{3} \\
\hline & & Conc & $10-50 \% \mathrm{~V} / \mathrm{V}$ & & & & \\
\hline & & Temp & $30^{\circ} \mathrm{C}$ and $60^{\circ} \mathrm{C}$ & & & & \\
\hline \multirow[t]{3}{*}{4.} & \multirow{3}{*}{$\begin{array}{l}\text { Aluminum/ } 0.5 \\
\mathrm{~mol} / \mathrm{L} \mathrm{HCl}\end{array}$} & \multicolumn{2}{|c|}{ Cocos nucifera $\mathrm{L}$. } & \multirow{3}{*}{$\begin{array}{l}\text { Amino acids, Ascorbic acid, } \\
\text { Vitamin B1,Sorbitol,Fructose, } \\
\text { Glucose,Malic acid, } \\
\text { Phytohormone,Auxin,Cytokinin, } \\
\text { Gibberellin, }\end{array}$} & \multirow{3}{*}{$\begin{array}{l}\text { Weight loss } \\
\text { Method }\end{array}$} & \multirow{3}{*}{$\begin{array}{l}\text { Spontaneous adsorption } \\
\text { IE }-93 \%\end{array}$} & \multirow[t]{3}{*}{4} \\
\hline & & Conc & $0-7.5 \% \mathrm{~V} / \mathrm{V}$ & & & & \\
\hline & & Temp & $30^{\circ} \mathrm{C}$ & & & & \\
\hline \multirow[t]{3}{*}{5.} & \multirow{3}{*}{$\begin{array}{l}\text { Aluminum alloy } \\
(7075) / \\
3.5 \% \mathrm{NaCl}\end{array}$} & \multicolumn{2}{|c|}{$\begin{array}{l}\text { Date palm (Phoenix } \\
\text { dactylifera L.) Fruit juice }\end{array}$} & \multirow[t]{3}{*}{$\begin{array}{l}\text { Cellobiose,Glucose,Xylose, } \\
\text { Fructose,Arabinose, }\end{array}$} & \multirow{3}{*}{$\begin{array}{l}\text { Potentiodynamic } \\
\text { polarization and } \\
\text { EIS }\end{array}$} & \multirow{3}{*}{$\begin{array}{l}\text { Physisorption } \\
\text { Cathodic } \\
\text { inhibitor }\end{array}$} & 5 \\
\hline & & Conc & $100-2000 \mathrm{ppm}$ & & & & \\
\hline & & Temp & -- & & & & \\
\hline 6. & Aluminium and & Garcin & dica & Garcinol,Curcumin & Tafel & Mixed-type inhibitor, & 6 \\
\hline & 6063 aluminium & Conc & $100-500 \mathrm{ppm}$ & & Extrapolation and & Chemisorption, & \\
\hline & $\begin{array}{l}\text { alloy/1.0 M } \\
\mathrm{H}_{3} \mathrm{PO}_{4}\end{array}$ & Temp & $30-50^{\circ} \mathrm{C}$ & & $\begin{array}{l}\text { Electrochemical } \\
\text { impedance } \\
\text { techniques, }\end{array}$ & $\begin{array}{l}\text { IE of Garcinia indica extract } \\
\text { was efficient for } 6063 \text { alloy } \\
\text { when compared with Al } \\
(99.6 \%)\end{array}$ & \\
\hline 7. & $\begin{array}{l}\text { Mild steel /1 M } \\
\mathrm{H}_{2} \mathrm{SO}_{4}\end{array}$ & $\begin{array}{l}\text { Arauce } \\
\text { Exudat }\end{array}$ & Columnaris (Gum & $\begin{array}{l}\text { Polysaccharide }(1,2- \\
\text { benzenedicarboxylic acid, }\end{array}$ & $\begin{array}{l}\text { Weight loss, } \\
\text { Electrochemical }\end{array}$ & $\begin{array}{l}\text { Maximum inhibition } \\
\text { efficiency is }\end{array}$ & 7 \\
\hline & & Conc & $50-600 \mathrm{ppm}$ & bis(2-ethylhexyl) ester, diisooctyl- & method, SEM & achieved at very lower & \\
\hline & & Temp & $303-323 \mathrm{~K}$ & $\begin{array}{l}\text { phthalate, } \\
\text { phthalic acid, isobutyl } \\
\text { and isopropyl ester) }\end{array}$ & & $\begin{array}{l}\text { concentration ( } 400 \mathrm{ppm}) \text {, } \\
\text { Mixed type inhibitor }\end{array}$ & \\
\hline
\end{tabular}




\begin{tabular}{|c|c|c|c|c|c|c|c|}
\hline \multirow{3}{*}{8.} & \multirow{3}{*}{$\begin{array}{l}\text { Aluminiumand } \\
\text { AA5754 } \\
\text { aluminium alloy/ } \\
3 \% \mathrm{NaCl}\end{array}$} & \multicolumn{2}{|c|}{ Laurus nobilis L.oil } & \multirow{3}{*}{$\begin{array}{l}\text { 1,8-cineole, } \\
\text { Methyl eugenol, } \\
\alpha \text {-terpinyl acetate, } \\
\text { Linalool, } \\
\text { Sabinene }\end{array}$} & \multirow{3}{*}{$\begin{array}{l}\text { Weight loss } \\
\text { method, } \\
\text { Potentiodynamic } \\
\text { polarization and } \\
\text { Linear } \\
\text { Polarization } \\
\text { method, SEM }\end{array}$} & \multirow{3}{*}{$\begin{array}{l}\text { AA5754 alloy has better } \\
\text { corrosion resistance in } 3 \% \\
\mathrm{NaCl} \text { solution than pure alloy }\end{array}$} & \multirow{3}{*}{8} \\
\hline & & \multicolumn{2}{|r|}{$0-50 \mathrm{ppm}$} & & & & \\
\hline & & Temp & $25^{\circ} \mathrm{C}$ & & & & \\
\hline \multirow[t]{3}{*}{9.} & \multirow{3}{*}{$\begin{array}{l}\text { Aluminum alloy } \\
(\mathrm{A} 17075) / \\
3.5 \% \mathrm{NaCl}\end{array}$} & \multicolumn{2}{|c|}{ L-Glutamine } & \multirow[t]{3}{*}{ Amino acid } & \multirow{3}{*}{$\begin{array}{l}\text { Polarization and } \\
\text { Electrochemical } \\
\text { impedance studies }\end{array}$} & \multirow{3}{*}{$\begin{array}{l}\text { The critical electrode rotation } \\
\text { rate for the best performance } \\
\text { of inhibitor is } 1000 \mathrm{rpm}\end{array}$} & \multirow[t]{3}{*}{9} \\
\hline & & Conc & $0-2000 \mathrm{rpm}$ & & & & \\
\hline & & Temp & -- & & & & \\
\hline \multirow[t]{3}{*}{10} & \multirow{3}{*}{$\begin{array}{l}\text { Aluminium/ } 0.5 \\
\mathrm{~mol} / \mathrm{L} \mathrm{HCl} . \\
\mathrm{KCl}, \mathrm{KBr} \text { are } \\
\text { added as additives }\end{array}$} & \multicolumn{2}{|c|}{ Morinda tinctoria leaves } & \multirow{3}{*}{$\begin{array}{l}\text { Alkaloids,Flavonoids, } \\
\text { Terpenoids,Steroids, } \\
\text { Amino acids }\end{array}$} & \multirow{3}{*}{$\begin{array}{l}\text { Mass loss and } \\
\text { electrochemical } \\
\text { techniques,SEM }\end{array}$} & \multirow{3}{*}{$\begin{array}{l}\text { IE - } 96.72 \% \\
\text { With immersion of } 2 \mathrm{~h} . \\
\text { Langmuir adsorption. } \\
\text { Physisorption }\end{array}$} & \multirow[t]{3}{*}{10} \\
\hline & & Conc & $0.5-7.0 \%$ & & & & \\
\hline & & Temp & $303-333 k$ & & & & \\
\hline \multirow[t]{3}{*}{11} & \multirow{3}{*}{$\begin{array}{l}\text { Aluminum \& } \\
\text { Aluminum silicon } \\
\text { alloy } / 0.5 \mathrm{M} \mathrm{HCl}\end{array}$} & \multicolumn{2}{|c|}{$\begin{array}{l}\text { Phoenix dactylifera } \\
\text { Leaves }\end{array}$} & \multirow{3}{*}{$\begin{array}{l}\text { Gallic acid, } \\
\text { Protocatechuic acid, Ferulic acid, } \beta \text { - } \\
\text { carotene, Lutein, Quercetin, } \\
\text { Luteiolin }\end{array}$} & \multirow{3}{*}{$\begin{array}{l}\text { Potentiodynamic } \\
\text { Polarization, EIS } \\
\& \text { EFM }\end{array}$} & \multirow[t]{3}{*}{$\begin{array}{l}\text { Mixed type Cathodic inhibitor, } \\
\text { Physisorption }\end{array}$} & \multirow[t]{3}{*}{11} \\
\hline & & Conc & $200-1000 \mathrm{ppm}$ & & & & \\
\hline & & Temp & $20^{\circ} \mathrm{C}$ & & & & \\
\hline \multirow[t]{3}{*}{12} & \multirow[t]{3}{*}{$\begin{array}{l}\text { Mild steel } \\
/ 1 \mathrm{M} \mathrm{HCl}\end{array}$} & \multicolumn{2}{|c|}{$\begin{array}{l}\text { Galinsoga parviflora (Quick } \\
\text { Weed) }\end{array}$} & \multirow[t]{3}{*}{$\begin{array}{l}\text { Alkaloids, Flavanoids, Saponins, } \\
\text { Tannins, Phytosterol, Galinsosides }\end{array}$} & $\begin{array}{l}\text { Weight loss, } \\
\text { Potentiodynamic }\end{array}$ & $\begin{array}{l}\text { IE - } 90 \% \text { at } 6 \% \mathrm{~V} / \mathrm{V}, \\
\text { Mixed-type inhibitor }\end{array}$ & 12 \\
\hline & & Conc & $2.5-6 \% \mathrm{~V} / \mathrm{V}$ & & polarization and & & \\
\hline & & Temp & $303-343 \mathrm{~K}$ & & & & \\
\hline 13 & Mild steel & Bombc & alabarieum seeds & Arachidic acid,Cyclopropane, & Weight loss, & $\mathrm{IE}-96.47 \%$ at $2.5 \% \mathrm{~V} / \mathrm{V}$, & 13 \\
\hline & $/ 1 \mathrm{M} \mathrm{HCl}$ & Conc & $\begin{array}{l}0.005 \%, 0.05 \% \\
0.15 \%, 0.5 \% \\
2.5 \%(\mathrm{~V} / \mathrm{V})\end{array}$ & $\begin{array}{l}\text { Lignoceric acid, Gossypol, Linoleic } \\
\text { acid,Myristic acid, Palmitic acid, } \\
\text { Oleic acid and Stearic acid }\end{array}$ & $\begin{array}{l}\text { Potentiodynamic } \\
\text { polarization } \\
\text { and EIS }\end{array}$ & Mixed-type inhibitor, & \\
\hline & & Temp & $303-343 \mathrm{~K}$ & & & & \\
\hline 14 & Copper/0.5 M & Myrtus & nmunis (oil extract) & Limonene, Linalool, & Weight Loss, & Mixed-type inhibitor & 14 \\
\hline & $\mathrm{Su}$ & Conc & $25-100 \mathrm{mg} / \mathrm{L}$ & $\begin{array}{l}\text { Pinene, 1,8-Cineole, Linanyl } \\
\text { Acetate, And Terpineol, }\end{array}$ & $\begin{array}{l}\text { Potentiodynamic } \\
\text { Polarization, EIS, }\end{array}$ & $\begin{array}{l}\text { affecting both the Cathodic } \\
\text { and anodic reactions }\end{array}$ & \\
\hline & & Temp & - & & SEM \& AFM & & \\
\hline 15 & $\begin{array}{l}\text { Copper/2.5 M } \\
\mathrm{HNO}_{3}\end{array}$ & $\begin{array}{l}\text { Vitami } \\
\text { (Thian }\end{array}$ & $\begin{array}{l}\text { hydrochloride } \\
\text { Hydrochloride) }\end{array}$ & -- & Weight loss study & $\begin{array}{l}\text { IE- } 69.9 \% \text { at } 2 \mathrm{mM} \text { thiamine } \\
\text { hydrochloride }\end{array}$ & 15 \\
\hline & & Conc & $0.01-2.5 \mathrm{mM}$ & & & & \\
\hline & & Temp & $\begin{array}{l}30,35,40 \& 45 \pm \\
0.2^{\circ} \mathrm{C}\end{array}$ & & & & \\
\hline
\end{tabular}


IRA-International Journal of Applied Sciences

\begin{tabular}{|c|c|c|c|c|c|c|c|}
\hline \multirow[t]{3}{*}{16} & \multirow[t]{3}{*}{\begin{tabular}{|l} 
AISI steel \\
$4130 / 1 \mathrm{M} \mathrm{HCl}$
\end{tabular}} & \multicolumn{2}{|c|}{$\begin{array}{l}\text { Taurine (2- } \\
\text { aminoethanesulfonic acid) }\end{array}$} & \multirow[t]{3}{*}{--} & \multirow{3}{*}{$\begin{array}{l}\text { Potentiodynamic } \\
\text { Polarization, } \\
\text { EIS,AFM \& } \\
\text { Optical } \\
\text { microscopy }\end{array}$} & \multirow[t]{3}{*}{$\begin{array}{l}\text { Mixed-type inhibitors, } \\
\text { Chemisorption }\end{array}$} & \multirow[t]{3}{*}{16} \\
\hline & & Conc & $12.5-250 \mathrm{ppm}$ & & & & \\
\hline & & Temp & $\begin{array}{l}25^{\circ} \mathrm{C}, 45^{\circ} \mathrm{C}, \\
65^{\circ} \mathrm{C} \\
\end{array}$ & & & & \\
\hline \multirow[t]{3}{*}{17} & \multirow[t]{3}{*}{$\begin{array}{l}\text { Carbon steel } \\
\text { (ASTM 1015)/ } \\
1.0 \mathrm{~mol} \mathrm{~L}^{-1} \mathrm{HCl}\end{array}$} & \multicolumn{2}{|c|}{$\begin{array}{l}\text { Antibacterial drugs (Penicillin } \\
\text { G, Ampicillin \& Amoxicillin } \\
\text { Drugs) }\end{array}$} & \multirow[t]{3}{*}{--} & \multirow{3}{*}{$\begin{array}{l}\text { Potentiodynamic } \\
\text { polarization, } \\
\text { EIS and } \\
\text { Electrochemical } \\
\text { noise }(\mathrm{EN})\end{array}$} & \multirow[t]{3}{*}{ Mixed-type inhibitor } & \multirow[t]{3}{*}{17} \\
\hline & & Conc & 0.1 to $10.0 \mathrm{mM}$ & & & & \\
\hline & & Temp & $25-55^{\circ} \mathrm{C}$ & & & & \\
\hline \multirow[t]{3}{*}{18} & \multirow[t]{3}{*}{$\begin{array}{l}\text { C38 Steel// } \\
1 \mathrm{M} \mathrm{HCl}\end{array}$} & \multicolumn{2}{|c|}{$\begin{array}{l}\text { Black pepper extract and its } \\
\text { piperine }\end{array}$} & \multirow[t]{3}{*}{$\begin{array}{l}\text { Piperine, } \\
\text { Piperanine }\end{array}$} & \multirow[t]{3}{*}{$\begin{array}{l}\text { Weight loss } \\
\text { method }\end{array}$} & \multirow[t]{3}{*}{ IE $-95.8 \%$ at $2 \mathrm{~g} / \mathrm{L}$} & \multirow[t]{3}{*}{18} \\
\hline & & Conc & $0.008-2 \mathrm{~g} / \mathrm{L}$ & & & & \\
\hline & & Temp & $40-70^{\circ} \mathrm{C}$ & & & & \\
\hline \multirow{3}{*}{19} & \multirow{3}{*}{$\begin{array}{l}\mathrm{C} 38 \text { steel/0.5 M } \\
\mathrm{H}_{2} \mathrm{SO}_{4}\end{array}$} & \multicolumn{2}{|c|}{ Lavandula multifida $\mathrm{L}$} & \multirow[t]{3}{*}{ Carvacrol } & \multirow{3}{*}{$\begin{array}{l}\text { Weight loss, } \\
\text { Electrochemical } \\
\text { Polarization \& } \\
\text { EIS methods }\end{array}$} & \multirow{3}{*}{$\begin{array}{l}\text { Mixed-type inhibitor, } \\
\mathrm{IE}-72.2 \% \text { at } 2 \mathrm{~g} / \mathrm{L} \text { of oil at } 298 \\
\mathrm{~K}\end{array}$} & \multirow[t]{3}{*}{19} \\
\hline & & Conc & $0.25-2 \mathrm{~g} / \mathrm{L}$ & & & & \\
\hline & & Temp & $303-343 \mathrm{~K}$ & & & & \\
\hline \multirow[t]{3}{*}{20} & \multirow{3}{*}{$\begin{array}{l}\text { Mild steel/ } 1 \mathrm{M} \\
\mathrm{HCl}\end{array}$} & \multicolumn{2}{|c|}{ Tagetes erecta stem } & \multirow{3}{*}{$\begin{array}{l}\beta \text {-caryophyllene }(8.5 \%), \\
\text { terpinolene }(18.4 \%),(\mathrm{E}) \text {-ocimenone } \\
(12.6 \%),(\mathrm{Z}) \beta \text {-ocimene }(10.4 \%), \\
\text { piperitenone }(10.4 \%),(\mathrm{Z})- \\
\text { ocimenone }(5.5 \%), \text { limonene }(6.2 \%)\end{array}$} & \multirow{3}{*}{$\begin{array}{l}\text { Chemical and } \\
\text { Electrochemical } \\
\text { studies, } \\
\text { FTIR }\end{array}$} & \multirow{3}{*}{$\begin{array}{l}\mathrm{IE}-97 \% \text { at } 0.3 \% \mathrm{~V} / \mathrm{V} \text {, } \\
\text { Mixed- type inhibitor }\end{array}$} & \multirow[t]{3}{*}{20} \\
\hline & & Conc & $0.001-0.3 \% \mathrm{~V} / \mathrm{V}$ & & & & \\
\hline & & Temp & $30^{\circ} \mathrm{C}$ & & & & \\
\hline 21 & Mild Steel/ & Acaci & es (Exudate gum) & Polysaccharides like $42 \%$ & Weight loss, & Mixed-type inhibitor, & 21 \\
\hline & $0.5 \mathrm{M}, 1 \mathrm{M}, 2 \mathrm{M}$ & Conc & $0.6 \mathrm{mg} / \mathrm{L}$ & Galactosyl, 27\% Arabinosyl, & Hydrogen & Inhibition efficiency increases & \\
\hline & $\mathrm{HCl}$ and $\mathrm{H}_{2} \mathrm{SO}_{4}$ & Temp & $30^{\circ} \mathrm{C}$ & $\begin{array}{l}15 \% \text { Rhamnosyl, } \\
14.5 \% \text { Glucuronoysl } \\
1.5 \% \text {-O-methyl-glucuronosyl and } \\
\text { Protein- polysaccharide (minor } \\
\text { component) }\end{array}$ & $\begin{array}{l}\text { evolution, } \\
\text { Electrochemical } \\
\text { polarization, } \\
\text { SEM,FTIR,XPS }\end{array}$ & $\begin{array}{l}\text { in the presence of magnetic } \\
\text { field }\end{array}$ & \\
\hline 22 & $\begin{array}{l}\text { Mild steel/ } 1 \mathrm{M} \\
\mathrm{HCl}\end{array}$ & $\begin{array}{l}\text { Alston } \\
\text { Var.la } \\
\text { Leave }\end{array}$ & $\begin{array}{l}\text { nugustifolia } \\
\text { ia(A. latifolia) }\end{array}$ & $\begin{array}{l}\text { Indole alkaloids (Alstogustine } \\
\text { 19-epialstogustine } \\
\text { Alstopirocine) }\end{array}$ & EIS,SEM,FTIR & $\begin{array}{l}\text { Mixed- type inhibitor, } \\
\text { IE-84\% for } \\
\text { Impedance studies, }\end{array}$ & 22 \\
\hline & & Conc & $1-5 \mathrm{mg} / \mathrm{L}$ & & & IE- $88 \%$ for & \\
\hline & & Temp & $30 \pm 2^{\circ} \mathrm{C}$ & & & Polarization studies & \\
\hline
\end{tabular}




\begin{tabular}{|c|c|c|c|c|c|c|c|}
\hline \multirow[t]{3}{*}{23.} & \multirow[t]{3}{*}{$\begin{array}{l}\text { Mild steel } / 0.5 \mathrm{M} \\
\mathrm{H}_{2} \mathrm{SO}_{4}\end{array}$} & \multicolumn{2}{|c|}{$\begin{array}{l}\text { Anacyclus pyrethrum } \mathrm{L} . \\
\text { (leaves and stem, } \\
\text { flowers,roots) }\end{array}$} & \multirow[t]{3}{*}{$\begin{array}{l}\text { Catechol tannins, } \\
\text { saponins, alkaloids, flavonoids } \\
\text { (absent in roots), amino acids }\end{array}$} & \multirow[t]{3}{*}{$\begin{array}{l}\text { EIS, } \\
\text { Potentiodynamic } \\
\text { Polarization }\end{array}$} & \multirow{3}{*}{$\begin{array}{l}\text { IE- } 87.01 \% \text { for leaves \& stems, } \\
88.88 \% \text { for flowers, and } \\
79.30 \% \text { for roots, } \\
\text { Chemisorption }\end{array}$} & \multirow[t]{3}{*}{23} \\
\hline & & Conc & $100-350 \mathrm{mg} / \mathrm{L}$ & & & & \\
\hline & & Temp & $25-40^{\circ} \mathrm{C}$ & & & & \\
\hline \multirow[t]{3}{*}{24} & \multirow{3}{*}{$\begin{array}{l}\mathrm{C} 38 \text { steel/ } 1 \mathrm{M} \\
\mathrm{HCl}\end{array}$} & \multicolumn{2}{|c|}{ Aniba rosaeodora } & \multirow[t]{3}{*}{ Anibine } & \multirow{3}{*}{$\begin{array}{l}\text { Potentiodynamic } \\
\text { polarization,EIS, } \\
\text { XPS, NMR }\end{array}$} & \multirow{3}{*}{$\begin{array}{l}\text { Mixed -type inhibitor, } \\
\text { Anibine as the major } \\
\text { constituent(EIS) }\end{array}$} & \multirow[t]{3}{*}{24} \\
\hline & & Conc & $25-200 \mathrm{mg} / \mathrm{L}$ & & & & \\
\hline & & Temp & $25^{\circ} \mathrm{C}$ & & & & \\
\hline \multirow[t]{3}{*}{25} & \multirow[t]{3}{*}{$\begin{array}{l}\text { Mild steel } \\
/ \mathrm{HCl}\end{array}$} & \multicolumn{2}{|c|}{$\begin{array}{l}\text { Areca catechu } \\
\text { (Seed extract) }\end{array}$} & \multirow{3}{*}{$\begin{array}{l}\text { polyphenols (flavonols } \\
\text { and tannins), alkaloids (Arecoline, } \\
\text { Arecaidine, } \\
\text { Guvacoline, and Guvacine), } \\
\text { carbohydrates, fats, proteins, } \\
\text { crude fiber, and mineral matters }\end{array}$} & \multirow{3}{*}{$\begin{array}{l}\text { Weight loss, } \\
\text { polarization \& } \\
\text { impedence } \\
\text { studies, } \\
\text { FTIR,SEM }\end{array}$} & \multirow[t]{3}{*}{ Mixed mode of inhibition } & \multirow[t]{3}{*}{25} \\
\hline & & Conc & $0-500 \mathrm{ppm}$ & & & & \\
\hline & & Temp & $303-318 \mathrm{~K}$ & & & & \\
\hline \multirow[t]{3}{*}{26} & \multirow{3}{*}{$\begin{array}{l}\text { Mild steel/ } 4 \mathrm{~N} \\
\mathrm{HCl} \& \mathrm{Conc} . \mathrm{HCl}\end{array}$} & \multicolumn{2}{|c|}{ Artemisia pallens } & \multirow{3}{*}{$\begin{array}{l}\text { Tetrahydrofuran, Davanone, } \\
\text { Linalool, Ethylcinnamate, } \\
\text { Sesquiterpenoids, Terpenoids, } \\
\text { Artemone }\end{array}$} & \multirow{3}{*}{$\begin{array}{l}\text { Weight loss, } \\
\text { Polarization } \\
4 \mathrm{~N} \mathrm{HCl,} \\
\text { Weight-loss, SEM } \\
\text { \& FTIR-Conc. } \\
\mathrm{HCl}\end{array}$} & \multirow{3}{*}{$\begin{array}{l}\mathrm{IE}-93 \% \text { at } 1.5 \mathrm{~g} / \mathrm{L} \text { in } 4 \mathrm{~N} \mathrm{HCl} \\
\mathrm{IE}-96.5 \% \text { at } 40 \mathrm{~g} / \mathrm{L} \text { in Conc. } \\
\mathrm{HCl}\end{array}$} & \multirow[t]{3}{*}{26} \\
\hline & & Conc & $0.1-1.5 \mathrm{~g} / 1$ & & & & \\
\hline & & Temp & $30 \pm 1{ }^{\circ} \mathrm{C}$ & & & & \\
\hline \multirow[t]{3}{*}{27} & \multirow[t]{3}{*}{$\begin{array}{l}\text { Mild steel/1 M } \\
\mathrm{HCl}\end{array}$} & \multicolumn{2}{|c|}{$\begin{array}{l}\text { Aquilaria crassna } \\
\text { Leaves extracts crude } \\
\text { extract(ME) and Aqueous } \\
\text { Extract(AE) }\end{array}$} & \multirow{3}{*}{$\begin{array}{l}\text { Saponins(higher presence), } \\
\text { Flavanoids \& Tannins(moderate } \\
\text { presence), } \\
\text { Alkaloids \& } \\
\text { Triterpenoids(Week presence) }\end{array}$} & \multirow[t]{3}{*}{$\begin{array}{l}\text { Weight loss, } \\
\text { Potentiodynamic } \\
\text { polarization, EIS, } \\
\text { XPS SEM,FTIR }\end{array}$} & \multirow{3}{*}{$\begin{array}{l}\text { Max IE at } 300 \text { ppm for ME } \\
80.28 \% \text { (Weight loss), } \\
66 \% \text { (EIS), } \\
67 \% \text { (Potentiodynamic } \\
\text { Polarization) and Max IE at } \\
60 \text { ppm for AE } 69.98 \% \\
\text { (weight loss), } 60 \% \\
\text { (Potentiodynamic } \\
\text { Polarization) } \\
\text { and } 59 \% \text { (EIS) at } 50 \mathrm{ppm}\end{array}$} & \multirow[t]{3}{*}{27} \\
\hline & & $\begin{array}{l}\text { Conc } \\
\text { (ME) } \\
\text { Conc } \\
\text { (AE) }\end{array}$ & $\begin{array}{l}0-300 \mathrm{ppm} \\
0-60 \mathrm{ppm}\end{array}$ & & & & \\
\hline & & Temp & $27{ }^{\circ} \mathrm{C}$ & & & & \\
\hline \multirow[t]{2}{*}{28} & \multirow{2}{*}{$\begin{array}{l}\text { Mild steel/1 N } \\
\mathrm{HCl}\end{array}$} & Basell & a L. leaves & Alkaloids,Carbohydrates, Pseudo & Weight loss, & Maximum & 28 \\
\hline & & Conc & $65-95 \mathrm{ppm}$ & tannins, Chlorogenic acids, & Potentiodynamic & inhibition efficiency was & \\
\hline
\end{tabular}


IRA-International Journal of Applied Sciences

\begin{tabular}{|c|c|c|c|c|c|c|c|}
\hline & & Temp & - & $\begin{array}{l}\text { Steroidal glycosides, Coumarin and } \\
\text { Flavonoids }\end{array}$ & $\begin{array}{l}\text { polarization, EIS, } \\
\text { SEM }\end{array}$ & $\begin{array}{l}81.28 \% \text { at the inhibitor } \\
\text { concentration } 95 \mathrm{ppm}\end{array}$ & \\
\hline \multirow[t]{3}{*}{29} & \multirow{3}{*}{$\begin{array}{l}\text { Mild steel/ } \\
1 \mathrm{M} \mathrm{HCl}\end{array}$} & \multicolumn{2}{|c|}{ Caulerpa racemosa } & \multirow{3}{*}{$\begin{array}{l}\text { Sesquiterpenes } \\
\text { Caulerpin an alkaloid } \\
\text { Reducing sugars } \\
\text { Galactose,glucose, arabinose } \\
\text { xylose, mannose, } \\
\text { rhamnose,amino acids }\end{array}$} & \multirow{3}{*}{$\begin{array}{l}\text { Weight loss, } \\
\text { EIS,UV,IR,NMR } \\
\text { \& AFM }\end{array}$} & \multirow{3}{*}{$\begin{array}{l}\text { Mixed-mode of inhibition } \\
\text { IE- } 83 \%\end{array}$} & \multirow[t]{3}{*}{29} \\
\hline & & Conc & $0-25 \mathrm{ppm}$ & & & & \\
\hline & & Temp & $303-323 \mathrm{~K}$ & & & & \\
\hline \multirow{4}{*}{30} & \multirow{4}{*}{$\begin{array}{l}\text { Mild steel/ } \\
0.5 \mathrm{M} \mathrm{H}_{2} \mathrm{SO}_{4}\end{array}$} & \multicolumn{2}{|c|}{ Centella asiatica (leaves) } & \multirow{4}{*}{$\begin{array}{l}\text { Triterpene acids (Asiatic, } \\
\text { Terminolic, Centic, } \\
\text { Centillic Centoic Acid) } \\
\text { Alkaloids(hydrocotylin), } \\
\text { volatile fatty oils(glycerites of } \\
\text { palmitic,stearic, lignoceric, } \\
\text { oleic,lynoleic and linolenic acids), } \\
\text { glycosides(asiaticoside A, } \\
\text { asiaticoside B, madecassoside, } \\
\text { centellic acid),and flavonoids(3- } \\
\text { glucosylquercetin, } \\
\text { 3-glucosylkaempferol and 7- } \\
\text { glucosylkaempferol }\end{array}$} & \multirow{4}{*}{$\begin{array}{l}\text { Gravimetric, } \\
\text { EIS,SEM }\end{array}$} & \multirow{4}{*}{$\begin{array}{l}\text { IE-95.08\% for } 1200 \mathrm{ppm} \text { at } \\
303 \mathrm{~K} \text {, } \\
\text { Mixed-type inhibitor }\end{array}$} & \multirow[t]{4}{*}{30} \\
\hline & & Conc & $0-1200 \mathrm{ppm}$ & & & & \\
\hline & & Temp & $30-60^{\circ} \mathrm{C}$ & & & & \\
\hline & & & & & & & \\
\hline \multirow[t]{3}{*}{31} & \multirow{3}{*}{$\begin{array}{l}\text { Mild steel/1 M } \\
\mathrm{HCl} \mathrm{\&} \\
0.5 \mathrm{M} \mathrm{H}_{2} \mathrm{SO}_{4}\end{array}$} & \multicolumn{2}{|c|}{$\begin{array}{l}\text { Chlorophytum borivilianum } \\
\text { root }\end{array}$} & \multirow[t]{3}{*}{ Saponins } & \multirow{3}{*}{$\begin{array}{l}\text { Weight loss, EIS, } \\
\text { SEM,FTIR,UV- } \\
\text { Visible } \\
\text { spectroscopy }\end{array}$} & \multirow[t]{3}{*}{ Mixed-type inhibitor } & \multirow[t]{3}{*}{31} \\
\hline & & Conc & $300-500 \mathrm{mg} / \mathrm{L}$ & & & & \\
\hline & & Temp & $35-55^{\circ} \mathrm{C}$ & & & & \\
\hline \multirow[t]{3}{*}{32} & \multirow[t]{3}{*}{$\begin{array}{l}\text { Mild steel/ } \\
1 \mathrm{M} \mathrm{HCl}\end{array}$} & \multicolumn{2}{|c|}{$\begin{array}{l}\text { Clematis gouriana } \\
\text { Leaves }\end{array}$} & \multirow[t]{3}{*}{$\begin{array}{l}\text { Magnoflorine, } \\
\text { Aporphine alkaloid, }\end{array}$} & \multirow{3}{*}{$\begin{array}{l}\text { Weight loss, } \\
\text { Potentiodynamic } \\
\text { polarization, } \\
\text { EIS,FTIR } \\
\text { SEM,EDS }\end{array}$} & \multirow{3}{*}{$\begin{array}{l}\text { IE-95.70\%. } \\
\text { Possibility of both } \\
\text { physisorption and } \\
\text { chemisorption }\end{array}$} & \multirow[t]{3}{*}{32} \\
\hline & & Conc & $0-400 \mathrm{ppm}$ & & & & \\
\hline & & Temp & $300-330 \mathrm{~K}$ & & & & \\
\hline \multirow[t]{3}{*}{33} & \multirow[t]{3}{*}{$\begin{array}{l}\text { Mild steel/1 M } \\
\mathrm{HCl} \& \mathrm{H}_{2} \mathrm{SO}_{4}\end{array}$} & \multicolumn{2}{|c|}{$\begin{array}{l}\text { Ervatamia coronaria } \\
\quad \text { (leaf extract) }\end{array}$} & \multirow[t]{3}{*}{$\begin{array}{l}\text { Ervatinine-An alkaloid,indole } \\
\text { Alkaloids,Tri-terpenoids }\end{array}$} & \multirow[t]{3}{*}{$\begin{array}{l}\text { Weight loss,EIS } \\
\text { SEM, XRD }\end{array}$} & \multirow[t]{3}{*}{ Mixed mode inhibitor } & \multirow[t]{3}{*}{33} \\
\hline & & Conc & $10-50 \mathrm{ppm}$ & & & & \\
\hline & & Temp & $303-323 \mathrm{~K}$ & & & & \\
\hline \multirow[t]{2}{*}{34} & Mild steel/ & Fig lea & & - & Weight loss & IE-87\% for concentration & 34 \\
\hline & $2 \mathrm{M} \mathrm{HCl}$ & Conc & $0-1000 \mathrm{ppm}$ & & Measurement, & $a b c$ & \\
\hline
\end{tabular}


IRA-International Journal of Applied Sciences

\begin{tabular}{|c|c|c|c|c|c|c|c|}
\hline & & Temp & $25 \pm 2^{\circ} \mathrm{C}$ & & $\begin{array}{l}\text { Electrochemical } \\
\text { techniques }\end{array}$ & Physisorption, & \\
\hline \multirow[t]{3}{*}{35} & \multirow[t]{3}{*}{$\begin{array}{l}\text { Mild steel/ } \\
2.5 \mathrm{M} \mathrm{H}_{2} \mathrm{SO}_{4}\end{array}$} & \multicolumn{2}{|c|}{$\begin{array}{l}\text { Gnetum Africana } \\
\text { (Ethanol extract) }\end{array}$} & \multirow{3}{*}{$\begin{array}{l}\text { Alkaloid,Saponin, Tannin,Terpene, } \\
\text { Anthraquinone,Flavonoid, } \\
\text { Cardiac glycoside, } \\
\text { Phlobatanins, Amino acids }\end{array}$} & \multirow{3}{*}{$\begin{array}{l}\text { Weight loss, } \\
\text { Gasometric, } \\
\text { Thermometric } \\
\text { methods, FTIR }\end{array}$} & \multirow[t]{3}{*}{ Physisorption } & \multirow[t]{3}{*}{35} \\
\hline & & Conc & $\begin{array}{ll}0.1 & 0.5 \mathrm{~g} / \mathrm{L} \\
\end{array}$ & & & & \\
\hline & & Temp & $303-333 \mathrm{~K}$ & & & & \\
\hline \multirow[t]{3}{*}{36} & \multirow[t]{3}{*}{$\begin{array}{l}\text { Mild steel/1M } \\
\mathrm{HCl} \& \mathrm{H}_{2} \mathrm{SO}_{4}\end{array}$} & \multicolumn{2}{|c|}{$\begin{array}{l}\text { Hydroclathrus clathratus } \\
\text { (marine alga) }\end{array}$} & \multirow{3}{*}{$\begin{array}{l}\mathrm{N} \text {-[2-hydroxy-1- } \\
\text { (hydroxymethyl)pentadecyl]eicosan } \\
\text { amide, Uracil, } \\
\text { 1-(2-deoxy-b-D-ribofuranosyl)-5- } \\
\text { methyl-1,2,3,4- } \\
\text { tetrahydropyrimidine-2,4-dione, and } \\
\text { 2-phenylamino-1,4-naphthoquinone }\end{array}$} & \multirow{3}{*}{$\begin{array}{l}\text { Weight loss, } \\
\text { EIS, SEM \& } \\
\text { AFM }\end{array}$} & \multirow{3}{*}{$\begin{array}{l}\text { Mixed-mode of inhibition, } \\
\text { IE-65.28\% for } 1 \mathrm{M} \mathrm{HCl} \\
\text { IE-77.64\% for } 1 \mathrm{M} \\
\mathrm{H}_{2} \mathrm{SO}_{4} \text { (Impedance studies) } \\
\text { IE-76.6\% for } 1 \mathrm{M} \mathrm{HCl} \\
\mathrm{IE}-85.02 \% \text { for } 1 \mathrm{M} \mathrm{H}_{2} \mathrm{SO}_{4} \\
\text { (Polarization studies) at } 500 \\
\text { ppm }\end{array}$} & \multirow[t]{3}{*}{36} \\
\hline & & Conc & $100-500 \mathrm{ppm}$ & & & & \\
\hline & & Temp & $303-323 K$ & & & & \\
\hline \multirow[t]{3}{*}{37} & \multirow{3}{*}{$\begin{array}{l}\text { Mild steel/1 M } \\
\mathrm{H}_{2} \mathrm{SO}_{4} \text { and } \\
1 \mathrm{M} \mathrm{HCl}\end{array}$} & \multicolumn{2}{|c|}{ Kigelia pinnata Leaves } & \multirow{3}{*}{$\begin{array}{l}\text { Saponins, Tannins, } \\
\text { Phenolic } \\
\text { acids, Flavonoids, } \\
\text { Alkaloids, and Carbohydrates }\end{array}$} & \multirow{3}{*}{$\begin{array}{l}\text { Weight loss,Tafel } \\
\text { polarization,EIS, } \\
\text { X-ray diffraction, } \\
\text { SEM,FTIR,UV- } \\
\text { Visible } \\
\text { Spectroscopy }\end{array}$} & \multirow{3}{*}{$\begin{array}{l}\text { The inhibitor inhibits the } \\
\text { corrosion reaction in both acid } \\
\text { environments and inhibition } \\
\text { efficiency follows the order } \\
\mathrm{H}_{2} \mathrm{SO}_{4}>\mathrm{HCl}\end{array}$} & \multirow[t]{3}{*}{37} \\
\hline & & Conc & $25-125 \mathrm{ppm}$ & & & & \\
\hline & & Temp & $\begin{array}{l}308 \mathrm{~K}, 313 \mathrm{~K}, 318 \\
\mathrm{~K}, 323 \mathrm{~K} \& 328 \\
\mathrm{~K}\end{array}$ & & & & \\
\hline \multirow[t]{3}{*}{38} & \multirow{3}{*}{$\begin{array}{l}\text { Mild steel/ } 1 \mathrm{M} \\
\mathrm{HCl} \text { and } 0.5 \mathrm{M} \\
\mathrm{H}_{2} \mathrm{SO}_{4}\end{array}$} & \multicolumn{2}{|c|}{ Syzygium cumini seed } & \multirow{3}{*}{$\begin{array}{l}\text { Ellagic acid, } \\
\text { Gallic acid, } \\
\text { Quercetin, } \\
\text { Cafeic acid }\end{array}$} & \multirow{3}{*}{$\begin{array}{l}\text { Weight loss, } \\
\text { EIS, }\end{array}$} & \multirow{3}{*}{$\begin{array}{l}\text { The inhibition efficiency is } \\
\text { slightly better in } \mathrm{HCl} \text { solution, } \\
\text { Mixed -type inhibitor }\end{array}$} & \multirow[t]{3}{*}{38} \\
\hline & & Conc & $60-600$ ppm & & & & \\
\hline & & Temp & $308-338 \mathrm{~K}$ & & & & \\
\hline \multirow[t]{3}{*}{39} & \multirow{3}{*}{$\begin{array}{l}6063 \text { Aluminium } \\
\text { alloy / } 1 \mathrm{M} \\
\text { phosphoric acid }\end{array}$} & \multicolumn{2}{|c|}{$\begin{array}{l}\text { Coriandrum sativum L. seed } \\
\text { extract }\end{array}$} & \multirow[t]{3}{*}{$\begin{array}{l}\text { Linalool } \\
\text { (Major constituent) }\end{array}$} & \multirow{3}{*}{$\begin{array}{l}\text { Potentiodynamic } \\
\text { polarization, } \\
\text { EIS,SEM }\end{array}$} & \multirow{3}{*}{$\begin{array}{l}\text { Mixed inhibitor, Results } \\
\text { obtained by Tafel polarisation } \\
\text { method and EIS method were } \\
\text { in good agreement with one } \\
\text { another. SEM studies } \\
\text { confirmed the corrosion } \\
\text { inhibition of the alloy due to } \\
\text { the adsorption of the inhibitor }\end{array}$} & \multirow[t]{3}{*}{39} \\
\hline & & Conc & $100-500$ ppm & & & & \\
\hline & & Temp & $30^{\circ} \mathrm{C}$ to $50^{\circ} \mathrm{C}$ & & & & \\
\hline \multirow[t]{3}{*}{40} & Carbon steel/ & Sesbar & sban & -- & Weight loss and & IE - 91.08\%, & 40 \\
\hline & & Conc & $0.2-2 \mathrm{~g} / \mathrm{l}$ & & Polarization & & \\
\hline & & Temp & $25^{\circ} \mathrm{C}$ to $65^{\circ} \mathrm{C}$ & & techniques & & \\
\hline
\end{tabular}




\begin{abstract}
Abbreviations:
NMR: Nuclear Magnetic Resonance Spectroscopy

EIS: Electrochemical Impedance Spectroscopy

AFM: Atomic Force Microscopy

SEM: Scanning Electron Microscopy

FTIR: Fourier Transform Infrared Spectroscopy

EDS: Energy Dispersive Spectrum

EFM: Electrochemical Frequency Modulation
\end{abstract}

\title{
Conclusion:
}

From the review article, it can be seen that the common phytoconstituents responsible for corrosion inhibition are saponins, alkaloids, flavonoids, and phenolic compounds. The major techniques adopted to evaluate the corrosion inhibition efficiency are : Weight -loss method, Electrochemical impedance studies, Thermometric method, Gasometric method. The surface morphology techniques used are scanning electron microscope (SEM), Energy - dispersive X-ray analysis (EDAX) and Fourier transform infra-red spectroscopy which confirms the formation of protective film on the corroded metal therein to stop the corrosion process.

All the plant materials have been evaluated as corrosion inhibitors in the laboratory level, unless it is being used in industries the research is not of much use. Hence transfer of technology is essential. These plant materials as corrosion inhibitors may be tested in various industries, where chemical inhibitors are already in use. To apply the plant materials as inhibitor in the industries the inhibitors should be standardized for which the reliability and reproducibility tests should be carried out. Hence more research could be carried out using plant material as corrosion inhibitors in the applied field.

\section{REFERENCES}

1.Ihebrodike M.Mejeha, Michael C.Nwandu, Kelechukwu B.Okeoma, Lebe A. Nnanna, Maduabuchi A. Chidiebere, Francis C. Eze, Emeka E.Oguzie, "Experimental and theoretical assessment of the inhibiting action of Aspilia africana extract on corrosion of aluminium alloy AA3003 in hydrochloric acid", J. Mater. Sci, (47) (2012) pp.2559-2572.

2. S.A.Umoren, U.M.Eduok, A.U.Israel, I.B.Obot, M.M.Solomon "Coconut coir dust extract: A novel eco-friendly corrosion inhibitor for $\mathrm{Al}$ in $\mathrm{HCl}$ solutions Green chemistry letters and reviews (5) (3) (2012) pp-303-313.

3. I.B.Obot, N.O.Obi-Egbedi "An interesting and efficient green corrosion inhibitor for aluminum from extracts of Chlomolaena odorata L.in acidic solution J.Appl Electrochem (40) (2010) pp-1977-1984.

4.Olusegun K.Abiola, Y.Tobun " Cocos nucifera $L$. as green corrosion inhibitor for acid corrosion of aluminum in $\mathrm{HCl}$ solution, Chinese Chemical letters (21)( 2010), pp-1449-1452.

5. Husnun Gerenji “Anticorrosive properties of Date palm (Phoenix dactylifera L.)Fruit juice on 7075 type aluminum alloy in $3.5 \% \mathrm{NaCl}$ solution; Industrial engineering chemistry research (51) (2012) pp$12835-12843$

6. Deepa Prabhu ,Padmalatha Rao "Adsorption and inhibition action of a novel green inhibitor on aluminium and 6063 aluminum alloy in $1.0 \mathrm{M} \mathrm{H}_{3} \mathrm{PO}_{4}$ solution" Procedia Materials Science (5)(2014 ), pp-222-231. 
7. Brindha $\mathrm{T}$, Revathi $\mathrm{P}$ and Mallika $\mathrm{J}$ "Corrosion Inhibition of Naturally Occurring Gum Exudates of Araucaria columnaris on Mild Steel in $1 \mathrm{M} \mathrm{H}_{2} \mathrm{SO}_{4}$ " International Research Journal of Environment Sciences Vol. 4(4), (2015) pp-36-43.

8.Jasna Halambek ,Katarina Berkovic ,Jasna Vorkapic-Furac " Laurus nobilis L.oil as green corrosion inhibitor for aluminum and AA5754 aluminum alloy in $3 \% \mathrm{NaCl}$ solution Materials chemistry and physics (137) (2013), pp-788-795.

9. H.Ashassi-Sorkhabi, E.Asghari "Electrochemical corrosion behavior of A17075 rotating disc electrode in neutral solution containing L-glutamine as a green inhibitor J.Appl Electrochemistry (40) (2010), pp631-637.

10. K.Krishnaveni, J.Ravichandran "Effect of aqueous extract of leaves of Morinda tinctoria on corrosion inhibition of aluminum surface in HCl medium", Trans. Nonferrous Met.Soc.China (24) (2014), pp-27042712

11.K.Shalabi, A.S.Fouda, G.Y.Elewady, A.El.Askalany " Adsorption and inhibitive properties of Phoenix dactylifera L. extract as a green inhibitor for aluminum and aluminum -silicon alloy in $\mathrm{HCl}$ ", Protection of metals and physical chemistry of surfaces (50) (3) (2014) pp-420-431.

12. P. Divya, R. Saratha and S.V. Priya "Impediment Effect of Galinsoga parviflora (Quick Weed) on Mild Steel Corrosion in 1 M HCl”, 2016, Chemical Science Review and Letters, (5)(18) (2016),115-126.

13. S.V.Priya \& R. Saratha, "Effect of Acidic Extract of Bombax Malabarieum as Green Corrosion Inhibitor for Mild Steel in $\mathrm{HCl}$ " Proceedings of International Conference on Renewable Energy and Sustainable Environment - RESE 15, Dr. Mahalingam College of Engineering and Technology, Pollachi642003, India. August 10-13, (2015), pp.176-184.

14. Mansoor Bozorg, Taghi shahrabi Farahani, Jaber Neshati, Zahra Chaghazardi, Ghodsi Mohammadi Ziarani, "Myrtus Communis as green inhibitor of copper corrosion in sulfuric acid", Ind.Eng.Chem.. Res (53) (2014), pp-4295-4303.

15.Olusegun K.Abiola, Moriamo O.John, Patric O.Asekunowo, Peter C.Okafor \& Oladele O.James, “O3[(4-amino -2-methyl-5-pyrimidinyl) methyl]-5-(2-hydroxylethyl -4-methyl thiazolium chloride hydrochloride as green corrosion inhibitor of copper in $\mathrm{HNO}_{3}$ solution and its adsorption characteristics", Green chemistry letters and reviews (4) (3) (2011) pp-273-279.

16. A .R. Hoseinzadeh, I.Danaee, M. H. Maddahy \& M. Rashvand Avei "Taurine as a green corrosion inhibitor for AISI 4130 steel alloy in Hydrochloric Acid solution", Chem.Eng.Comm (201) (2014) pp$380-402$.

17. Gh. Golestani, M. Shahidi, \& D.Ghazanfari, "Electrochemical evaluation of antibacterial drugs as environment-friendly inhibitors for corrosion of carbon steel in $\mathrm{HCl}$ solution", Applied surface science (308) (2014) pp-347-362.

18.M. Dahmani, A.Et.Touhami, S.S.Al-Deyab, B.Hammouti, A.Bouyanzer Corrosion inhibition of C38 steel in $1 \mathrm{M} \mathrm{HCl}$ : A comparative study of Black pepper extract and its isolated piperine, Int.J.Electrochem. Sci (5)( 2010 ) pp-1060-1069.

19.M. Znini, J. Paolini, L. Majidi, J.-M. Desjobert, J. Costa N. Lahhit, A. Bouyanzer "Evaluation of the inhibitive effect of essential oil of Lavandula multifida L., on the corrosion behavior of C38 steel in $0.5 \mathrm{M}$ $\mathrm{H}_{2} \mathrm{SO}_{4}$ medium", Res.Chem. Intermed (38) (2012) pp-669-683. 
20. R. Subha and R. Saratha Corrosion Mitigation of low Carbon Steel in Hydrochloric acid Medium by Tagetes erecta Stem Extract, Res J. Chem. Environ. Sci. (4) (2) (2016) pp.19-26.

21. M.A.Abu-Dalo, A.A.Othman, N.A.F.Al-Rawashdeh, Exudate gum from Acacia trees as green corrosion inhibitor for mild steel in acidic media Int.J.Electrochem. Sci (7) (2012) pp-9303-9324.

22.Pandian Bothi Raja, Ahmad Kaleem Qureshi, Afidah Abdul Rahim, Khalijah Awang, Mat Ropi Mukhtar, and Hasnah Osman "Indole alkaloids of Alstonia anugustifolia Var.latifolia as green inhibitor for Mild steel corrosion in $1 \mathrm{M} \mathrm{HCl}$ media" Journal of Materials Engineering and performance,(22) (2013) pp-1072-1078.

23. Omar Benali, Chaouki Selles R.Salghi, "Inhibition of acid corrosion of mild steel by Anacyclus pyrethrum L. extracts", Res. Chem. Intermed. (40)(2014) pp-259-268.

24. Maxime chevalier, Florent Robert, Nadine Amusant Michel Traisnel, Christophe Roos Mounim Lebrini, "Enhanced corrosion resistance of mild steel in $1 \mathrm{M}$ hydrochloric acid solution by alkaloids extract from Aniba rosaeodora plant: Electrochemical, phytochemical and XPS studies", Electrochimica Acta (131) (2014) pp-96-105.

25.K.P. Vinod Kumar, M. Sankara Narayanan Pillai, G. Rexin Thusnavis, " Green corrosion inhibitor from seed extract of Areca catechu for Mild steel in hydrochloric acid medium”, J. Mater. Sci (46) (2011 )pp-5208-5215.

26.Patchaiah Kalaiselvi, Subbiah Chellammal, Seeni Palanichamy, Gopalan Subramanian, "Artemisia pallens as corrosion inhibitor for mild steel in $\mathrm{HCl}$ medium", Materials Chemistry and Physics (120) (2010) pp-643-648.

27.L.Y. S. Helen, A.A.Rahim, B.Saad, M.I. Saleh, P. Bothi Raja, “Aquilaria crassna leaves extracts-a green corrosion inhibitor for Mild steel in $1 \mathrm{M} \mathrm{HCl} \mathrm{medium",} \mathrm{Int.J.Electrochem.} \mathrm{Sci,} \mathrm{(9)} \mathrm{(2014)} \mathrm{pp-830-}$ 846.

28. J. Rosaline Vimala*, P. Priyadharshini and R. Helanthin Prasanthi, "Basella alba L. Extract as Corrosion Inhibitor for Mild Steel in Acid Medium, World Journal of Pharmacy and Pharmaceutical Sciences, (5) (3) (2016) pp.1704-1713.

29. Chennappan Kamal \& Mathur Gopalakrishnan Sethuraman, "Caulerpin-A bis-Indole Alkaloid as a green inhibitor for the corrosion of Mild steel in $1 \mathrm{M} \mathrm{HCl}$ solution from the Marine Alga Caulerpa racemosa", Ind.Eng.Chem.Res.(51) (2012) pp.10399-10407.

30. S.S.Shivakumar, K.N.Mohana, "Centella asiatica extracts as a green corrosion inhibitor for mild steel in 0.5 M Sulphuric Acid medium", Advances in Applied sciences Research (3)(5)(2012) pp.3097-3106.

31.Gopal Ji, Priyanka Dwivedi, Shanthi Sundaram and Rajiv Prakash, "Inhibitive effect of Chlorophytum borivilianum root extract on mild steel corrosion in $\mathrm{HCl}$ and Sulphuric Acid solutions", Ind.Eng.Chem Res.(52) (2013) pp.10673-10681.

32.Gopiraman Mayakrishnan, Sakunthala Pitchai, Kanmani Raman, Alex Ramani Vincent, Sulochana Nagarajan, Inhibitive action of Clematis gouriana extract on the corrosion of mild steel in acidic medium Ionics (17) 2011 pp-843-852.

33. M.G. Sethraman, V. Aishwarya, C. Kamal, T.Jebakumar Immanuel Edison, "Studies on ErvatinineThe anticorrosive phytoconstituent of Ervatamia coronaria", Arabian Journal of Chemistry (2013). 
34. Taleb H. Ibrahim and Mohamed Abou Zour .Corrosion inhibition of Mild steel using Fig leaves extracts in Hydrochloric acid solution Int.J.Electrochem. Sci.(6) ( 2011) pp-6442-6455.

35.Nnabuk O.Eddy, Steven A.Odoemelam, Anduang O.Odiongenyi Inhibitive, adsorption and synergistic studies on ethanol extract of Gnetum Africana as green corrosion inhibitor for mild steel in $\mathrm{H}_{2} \mathrm{SO}_{4}$, Green chemistry letters and reviews, 2, (2)(2009) pp-111-119.

36. C. Kamal and M.G.Sethuraman, "Hydroclathrus clathratus marine alga as a green inhibitor of acid corrosion of mild steel” Res. Chem. Intermed, (39) (2013) pp-3813-3828.

37. P.Muthukrishnan, K.Saravana Kumar, B.Jeyaprabha and P. Prakash, "Anticorrosive activity of Kigelia pinnata leaves extract on Mild steel in acidic media", Metallurgical and Materials Transactions A, (45A) (2014) pp - 4510-4524.

38. Ambrish Singh, M.A.Quraishi, "The extract of Jamun (Syzygium cumini) seed as green corrosion inhibitor for acid media", Res.Chem. Intermed. (2013).

39. Deepa Prabhu and Rao Padmalatha, "Corrosion inhibition of 6063 aluminum alloy by Coriandrum sativum L seed extract in phosphoric acid medium”, J. Mater. Environ. Sci. 4 (5) (2013), pp. 732-743.

40. Hussein H. Al-Sahlanee, Abdul-Wahab A. Sultan and Mustafa M. Al-Faize, Corrosion Inhibition of Carbon Steel in 1M HCl Solution Using Sesbania Sesban Extract, Aquatic Science and Technology,(1) (2) (2013), pp.135-151. 